CLINICAL STUDY

\title{
An observational study of the initial management of hypothyroidism in France: the ORCHIDÉE study
}

\author{
Brigitte Delemer, Jean-Pierre Aubert ${ }^{1}$, Pierre $\mathrm{Nys}^{2}$, Frédéric Landron ${ }^{3}$ and Stéphane Bouée ${ }^{4}$ \\ Endocrinology, Hôpital Robert Debré, 45 Rue Cognacq Jay, 51100 Reims, France, ${ }^{1}$ General Medicine, Paris-Diderot University, Paris, France, \\ ${ }^{2}$ Endocrinology and Diabetes, Hospital and Private Practice, 11-13 rue Guersant, Paris, France, ${ }^{3}$ General Medicine and Specialities, Merck Serono S.A.S. \\ Lyon, 37 rue saint Romain, Lyon, France and ${ }^{4}$ Epidemiology, \& Statistics, Cemka-Eval, Bourg la Reine, France \\ (Correspondence should be addressed to B Delemer; Email: bdelemer@chu-reims.fr)
}

\begin{abstract}
Objective: To document the initial management of hypothyroidism in France with respect to diagnostic setting, investigations, and therapeutic approach.

Design: Observational study of the management by primary care practitioners (PCPs) and endocrinologists of patients diagnosed with, and treated for, hypothyroidism during the enrollment period or the previous 6 months.

Methods: A representative sample of PCPs and endocrinologists enrolled up to five consecutive patients and reported sociodemographic, clinical, therapeutic, and laboratory data. Data were submitted at baseline and at the first measurement of TSH after starting the treatment.

Results: The analysis population comprised 1255 patients (mean (s.D.) age 52.8 (16.3) years; 84\% female). Hypothyroidism was suspected on clinical grounds in $77 \%$ of patients, with goiter in $16 \%$. Autoimmune thyroiditis, supported by positive anti-thyroid antibodies, was the most frequent diagnosis $(59 \%)$, followed by iatrogenic causes $(28 \%)$, of which thyroidectomy was the most common. The median baseline TSH was $8.6 \mathrm{mIU} / \mathrm{l}$, suggesting a high incidence of subclinical hypothyroidism. Imaging studies were requested in over $75 \%$ of patients, with ultrasound performed in $98 \%$ and scintigraphy performed in $19 \%$ of these patients. Both groups of physicians treated their patients almost exclusively with levothyroxine. Endocrinologists were more likely than PCPs to provide counseling on how to take medication correctly.

Conclusions: This observational study of a large cohort of patients with newly diagnosed hypothyroidism in France illustrates current practice and indicates some areas where physician education may be required to optimize adherence to guidelines and cost-effectiveness.
\end{abstract}

European Journal of Endocrinology 167 817-823

\section{Introduction}

Hypothyroidism is a common condition, which varies in degree from asymptomatic to life-threatening and is usually secondary to thyroid disease. Clinical suspicion of hypothyroidism is easily confirmed by elevated plasma levels of TSH and low plasma levels of free thyroxine $\left(\mathrm{FT}_{4}\right)(1,2)$. In some patients (usually asymptomatic), TSH is elevated, but $\mathrm{FT}_{4}$ and free triiodothyronine levels are within the normal range; this state of compensated thyroid failure is commonly referred to as subclinical hypothyroidism $(3,4)$ and treatment of this condition is debated.

The most common cause of primary hypothyroidism in developed countries is autoimmune thyroiditis; other major causes include consequences of surgical thyroidectomy, administration of radioactive iodine or anti-thyroid drugs, and adverse effects of drugs given for conditions not related to the thyroid gland, such as amiodarone, lithium, and interferon $(5,6,7,8,9,10)$. In a cross-sectional study of the general population in Denmark, the prevalences of diagnosed, undiagnosed, and subclinical hypothyroidism, respectively, were 1.0, 0.4 , and $0.7 \%$ (9). In a longitudinal UK study using a randomly selected population sample, an incidence of $0.35 \% /$ year was seen for spontaneous hypothyroidism in women, which increased to $0.41 \%$ for all-cause hypothyroidism; the incidence of all-cause hypothyroidism in men was $0.06 \%$ (10). A French study that excluded patients with previous or current thyroid disease and those currently receiving thyroid hormones or anti-thyroid medication indicated that primary hypothyroidism was seen in $0.4 \%$ of women aged $35-44$ years, $0.7 \%$ of women aged $45-60$ years, and 
$0.2 \%$ of men aged 45-60 years (11); subclinical hypothyroidism was seen in $7.2,11.1$, and $4.0 \%$ of these populations respectively.

Adult patients typically require $\sim 1.7 \mu \mathrm{g} / \mathrm{kg}$ per day $\left(120 \mu \mathrm{g} /\right.$ day for a $70 \mathrm{~kg}$ adult) of levothyroxine $\left(\mathrm{L}-\mathrm{T}_{4}\right)$ for full hormone replacement (12). In older patients (aged $\geq 65$ years) or those with a history of cardiac disease, an initial low dose $(25 \mu \mathrm{g} /$ day $)$ should be titrated up only every 8 weeks (12).

Local consensus guidelines for the management of primary hypothyroidism are available in various countries, including France (13), but little is known about how local daily clinical practice relates to such guidelines. The Observatoire de la Prise en Charge Initiale de l'hypothyroidie en France (ORCHIDÉE) study was designed to describe the initial approaches to the management of hypothyroidism taken by primary care practitioners (PCPs) and endocrinologists in France, including diagnosis, treatment strategies, and first follow-up. Furthermore, the characteristics of a large population of de novo hypothyroid patients in France are described for the first time in this study.

\section{Materials and methods}

\section{Study design}

ORCHIDÉE (Merck Serono protocol no. 2007-106) was an observational study of patients who were starting treatment with thyroid hormones for hypothyroidism or had started therapy in the previous 6 months. Enrollment for this study was from 30 October 2008 to 5 February 2009. The primary objective was to document the details of the initial management of hypothyroidism, including circumstances of diagnosis, diagnostic work-up, and details of the treatment approach taken by the PCP or endocrinologist. The study was designed to record, but not to modify, the physicians' usual patient management practice and prescription habits.

Patients were recruited by a sample of 500 officebased PCPs in private practice and 120 endocrinologists who were office-based, hospital-based, or both (mixed). Over the course of a 3-month enrollment period, physicians were expected to enroll up to five consecutive eligible patients with hypothyroidism.

Sociodemographic, clinical, therapeutic management, and laboratory data were collected using a standardized case record form at baseline and at follow-up, which was taken to be the time of the result from the first TSH determination after starting the treatment with thyroid hormones.

This study was approved by the Conseil National de l'Ordre des Médecins (CNOM), the Comité Consultatif sur le Traitement de l'Information en matière de Recherche dans le domaine de la Santé (CCTIRS), and the
Commission Nationale de l'Informatique et des Libertés (CNIL). All patients gave verbal informed consent.

\section{Physician recruitment}

Lots were drawn from a physician database (Centre d'Etude de Gestion et de Documentation Informatique et de Marketing) to randomly select physicians to be invited to participate. This database is widely used in France to identify potential investigators for pharmacoepidemiological surveys. Selected physicians were sent a letter explaining the purpose of the research and a reply coupon on which they could indicate their interest.

\section{Patient selection}

Patients diagnosed with and treated for hypothyroidism by the recruited physician or by another physician during the enrollment period or during the previous 6 months were eligible for inclusion, provided that diagnostic data were available. Exclusion criteria included participation in a clinical trial within the last 3 months (including current participation), a strong likelihood of nonavailability for follow-up, short life expectancy, or previous problems with study participation.

\section{Analyses}

For this observational study, the number of patients to be recruited was based on the proportion of patients expected in subcategories (e.g. elderly patients, patients with coronary artery disease or arrhythmias), and on the size of the confidence intervals of proportions calculated in these subcategories. The two following formulas were used for the confidence interval calculation and for the number of patients to be included respectively (14):

$$
\begin{aligned}
& \mathrm{IC}_{95 \%}=p \pm 1,96 \sqrt{\frac{p \times q}{n}} \\
& n=1,96^{2} \times \frac{p \times q}{\text { required_precision }}{ }^{2}
\end{aligned}
$$

Accordingly, it was estimated that a population of 1000 patients would be sufficient to meet the study objectives.

Comparative analyses were performed for enrollment by PCP vs endocrinologist, TSH levels at study entry $(<0.2,0.2-0.4$, and $>4 \mathrm{mIU} / \mathrm{l})$, and etiology of hypothyroidism. Statistical analyses were performed using the SAS software package, version 9.1 (SAS Institute, Cary, NC, USA). For qualitative variables, Pearson's $\chi^{2}$ test was used unless the theoretical (calculated) sample size was $<5$, in which case Yates's continuity correction or Fisher's exact test was used. For quantitative variables, Student's $t$-test or ANOVA was used. 


\section{Results}

\section{Physician and patient recruitment}

Agreement to participate was secured from 500 of 5200 PCPs and 120 of 600 endocrinologists invited to participate. Of these, $254(50.8 \%)$ PCPs and 82 $(68.3 \%)$ endocrinologists enrolled at least one patient. The distribution of PCPs who enrolled at least one patient was comparable with that of the sample of invited PCPs by age, sex, and geographic region of practice but included a lower proportion of female physicians than the total PCP population in France (16.1 vs $31.7 \%$ ). The endocrinologist population was representative of all endocrinologists in France, except that hospital-based physicians were under-represented in the population who enrolled at least one patient (14.6\%) compared with all endocrinologists invited to participate $(52.4 \%)$. An analysis of this variable (hospital-based, office-based, and mixed) revealed hardly any differences between the groups.

Of 1284 patients enrolled in the study, 1255 were included in the analysis population (PCPs, $n=835$; endocrinologists, $n=420$ ). The 29 enrolled patients who were excluded did not meet the inclusion criteria because of a diagnosis of hypothyroidism $>6$ months before the study $(n=24)$, or other reasons $(n=5)$.

\section{Patient and disease characteristics}

Patient demographic characteristics and diagnostic details are shown in Table 1; patients enrolled by PCPs were older than those enrolled by endocrinologists $(P<0.001)$. Clinical suspicion of hypothyroidism was the reason for evaluation of thyroid function in about three-quarters of patients.

The clinical features of hypothyroidism reported most frequently were fatigue $(n=1007,80.2 \%)$, weight gain $(n=604,48.1 \%)$, gastrointestinal symptoms $(n=393$, $31.3 \%)$, cold intolerance $(n=376,30.0 \%)$, muscular symptoms $(n=312,24.9 \%)$, and skin changes $(n=281$, $22.4 \%)$. Cardiovascular risk factors recorded were hypertension $(n=332,26.4 \%)$, hypercholesterolemia ( $n=346,27.6 \%)$, history of smoking $(n=272,21.7 \%)$, and diabetes $(n=80,6.4 \%)$.

\section{Pretreatment laboratory results}

The results of TSH tests performed before prescription of thyroid hormone therapy were available for 1125 of the 1255 patients included in the analysis $(89.6 \%)$ (Table 2). Of the 130 patients in whom TSH results were not reported, TSH was not measured in 57 patients who were being treated following anti-thyroid therapy (surgical, pharmacological, or radioisotope). For the

Table 1 Patient demographics at baseline and circumstances of diagnosis of hypothyroidism.

\begin{tabular}{|c|c|c|c|}
\hline & $\begin{array}{l}\text { PCPs } \\
(n=835)\end{array}$ & $\begin{array}{l}\text { Endocrinologists } \\
(n=420)\end{array}$ & Total $(n=1255)$ \\
\hline \multicolumn{4}{|l|}{ Gender } \\
\hline Female (\%) & 83.9 & 85.2 & 84.4 \\
\hline \multicolumn{4}{|l|}{ Age (years) } \\
\hline Mean (S.D.) & $54.4(16.1)$ & $49.6(16.2)^{*}$ & $52.8(16.3)$ \\
\hline Median (range) & $54(10-104)$ & $50(11-89)$ & $52(10-104)$ \\
\hline \multicolumn{4}{|l|}{ BMI $\left(\mathrm{kg} / \mathrm{m}^{2}\right)$} \\
\hline \multirow{2}{*}{\multicolumn{4}{|c|}{ Circumstances of hypothyroidism diagnosis ${ }^{\mathrm{a}}, n(\%)$}} \\
\hline & & & \\
\hline Clinical suspicion of hypothyroidism & $673(80.6)$ & $288(68.6)^{*}$ & $961(76.6)$ \\
\hline Detection of goiter & $157(18.8)$ & $40(9.5)^{\star}$ & $197(15.7)$ \\
\hline Patients with pre-existing thyroid disease & $141(16.9)$ & $121(28.8)^{\dagger}$ & $262(20.9)$ \\
\hline Thyroid nodule & 55 & 27 & 82 \\
\hline Euthyroid goiter ${ }^{\mathrm{b}}$ & 29 & 20 & 49 \\
\hline Thyroid cancer ${ }^{\mathrm{C}}$ & 9 & 12 & 21 \\
\hline Hyperthyroidism & 34 & 43 & 77 \\
\hline Combination of reasons & 4 & 2 & 6 \\
\hline Other diseases & 9 & 15 & 24 \\
\hline Not recorded & 1 & 2 & 3 \\
\hline Other diagnostic circumstances & $45(5.4)$ & $52(12.4)^{*}$ & $97(7.7)$ \\
\hline latrogenic cause $\mathrm{d}^{\mathrm{d}}$ & 23 & 28 & 51 \\
\hline Systematic work-up & 13 & 15 & 28 \\
\hline Family history & 5 & 6 & 11 \\
\hline Other & 3 & 3 & 6 \\
\hline Not recorded & 1 & - & 1 \\
\hline
\end{tabular}

${ }^{\star} P<0.001$ compared with primary care practitioners (PCPs); ${ }^{\dagger} P<0.05$ compared with PCPs.

${ }^{a}$ More than one response could be given for each patient; no information was provided in 23 patients (PCPs, $n=18$; endocrinologists, $n=5$ ).

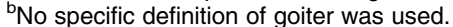

${ }^{\mathrm{C}}$ Radical treatment undertaken within the eligibility period.

${ }^{\mathrm{d}}$ No specific iatrogenic cause was notified. 
Table 2 Pretreatment laboratory assessments.

\begin{tabular}{lcc}
\hline & $\begin{array}{c}\text { Autoimmune } \\
\text { thyroiditis } \\
(n=739)\end{array}$ & $\begin{array}{c}\text { Total patient } \\
\text { population } \\
(n=1255)\end{array}$ \\
\hline TSH & & \\
Data available, $n(\%)$ & $718(97.2)$ & $1125(89.6)$ \\
Mean (s.D.), mIU/l & $25.8(118.4)$ & $23.4(97.6)$ \\
Median (range), mIU/I & $8.6(0.2-2768)$ & $8.6(0-2768)$ \\
Free thyroxine & & \\
Data available, $n(\%)$ & $433(58.6)$ & $665(53.0)$ \\
Mean (s.D.), pmol/l & $9.3(7.7)$ & $9.5(8.9)$ \\
Median (range), pmol/l & $9.0(0.1-90.3)$ & $9.0(0.1-125.1)$ \\
Anti-thyroperoxidase & & \\
antibodies & & \\
Data available, $n(\%)$ & $568(76.9)$ & $757(60.3)$ \\
Positive (\%) & $493(86.8)$ & $526(69.5)$ \\
Anti-thyroglobulin & & \\
antibodies & & $605(48.2)$ \\
Data available, $n(\%)$ & $452(61.2)$ & $369(61.0)$ \\
Positive (\%) & $348(77.0)$ & \\
Imaging studies, $n(\%)$ & & \\
Ultrasound & & \\
Scintigraphy & $98(86.7)$ & $(74.6)$ \\
\hline
\end{tabular}

remaining 73 patients, no adequate response was given on the case report form regarding whether or not TSH had been measured or TSH measurements were submitted.

There was a considerable inter-patient variation in TSH levels, which were $>4.0 \mathrm{mIU} / \mathrm{l}$ in the great majority of patients (1066/1125; 94.7\%), but $<10 \mathrm{mIU} / \mathrm{l}$ in over $50 \%$. Excluding patients with causes of hypothyroidism other than autoimmune thyroiditis had little effect on the calculated levels (Table 2).

Pretreatment $\mathrm{FT}_{4}$ levels were available in 678 (54\%) patients; of these, 601 (88.6\%) had a TSH level $>4.0 \mathrm{mIU} / \mathrm{l}$. Among these 601 patients who had TSH levels within the hypothyroid range, 210 (34.9\%) had pretreatment $\mathrm{FT}_{4}$ levels $>10.6 \mathrm{pmol} / \mathrm{l}$, indicating the presence of subclinical hypothyroidism.

\section{Thyroid imaging}

Imaging studies were requested in 956 patients (76.2\%). Thyroid ultrasound was performed in $97.9 \%$ of these patients and scintigraphy in $18.8 \%$, suggesting that both techniques were employed in almost $20 \%$ of these patients.

\section{Etiology of hypothyroidism}

The underlying cause of hypothyroidism was autoimmune thyroiditis in $59.4 \%$ of the 1245 patients for whom accurate causal information was provided. Iatrogenic causes were reported in 344 of these patients $(27.6 \%)$. Within this group, $52.9 \%(n=182)$ patients had been treated with surgery. Other iatrogenic causes were radioactive iodine $(n=30,8.7 \%)$, anti-thyroid drugs $(n=34,9.9 \%)$, combinations of anti-thyroid modalities $(n=10,2.9 \%)$, and 'other iatrogenic causes' $(n=88,25.6 \%)$, most $(n=78,88.6 \%)$ of which were treatment with amiodarone. The most common causes of hypothyroidism were similar for both physician groups.

\section{Therapeutic management}

Thyroid hormone therapy was initiated by the enrolling physician in $75 \%$ of cases. $\mathrm{L}^{-\mathrm{T}_{4}}$ (as tablets) was prescribed in almost all $(98.3 \%)$ patients, at a mean (s.D.) starting dose of 56.0 (36.0) $\mu \mathrm{g} /$ day by PCPs and 59.0 (35.6) $\mu \mathrm{g} /$ day by endocrinologists. Half of the patients started treatment at $<50 \mu \mathrm{g} /$ day, with successive titrations to mean doses of 71 and $83 \mu \mathrm{g} /$ day respectively. The mean interval between dose titrations was 6 weeks (median 4 weeks).

\section{Follow-up monitoring}

Follow-up data on TSH levels were reported in $94.4 \%$ of the patients in whom any follow-up data were submitted $(n=1208)$, usually at $2-3$ months after the initiation of treatment. At follow-up, mean (s.D.) TSH levels were 6.8 (36.1) $\mathrm{mIU} / \mathrm{l}$ and the median TSH level was $3.0 \mathrm{mIU} / \mathrm{l}$. Levels of $\mathrm{FT}_{4}$ at follow-up were available in $49.8 \%$ of patients and had increased to the lower end of the normal range (mean (S.D.), 12.4 (11.0) pmol/l; median, $12.8 \mathrm{pmol} / \mathrm{l})$.

The mean interval between TSH assessment and the start of thyroid replacement therapy was longer in patients with normal baseline TSH levels than in patients with high TSH levels (29.8 vs 16.3 days) (Table 3). Low or normal baseline TSH levels $(<0.2$ or $0.2-4 \mathrm{mIU} / \mathrm{l})$ were measured most commonly in patients with prior surgical, pharmacological, or radioisotope treatment for hyperthyroidism (40.7, 11.9 , and $5.1 \%$ respectively). Thyroid antibodies were more likely to be determined in patients with high baseline TSH (56-67\%) than in patients with low or normal baseline TSH (31-49\%), and the results were more likely to be positive (Table 3 ).

\section{Differences in management by etiology of hypothyroidism}

Most patients $(83.7 \%)$ with suspected hypothyroidism had baseline TSH levels above $4.0 \mathrm{mIU} / \mathrm{l}$ (Table 3). Patients with iatrogenic hypothyroidism were less likely to have TSH determined at baseline than patients with autoimmune thyroiditis or patients with other etiologies (63.8, 99.0, and 99.2\% respectively; $P<0.001)$. In 57 of the 89 patients with iatrogenic hypothyroidism in whom TSH was not determined, a history of thyroidectomy or treatment with anti-thyroid drugs was given as the reason. Patients with iatrogenic hypothyroidism were also less likely to have $\mathrm{FT}_{4}$ levels measured prior to treatment than patients with autoimmune thyroiditis or other causes (43.3, 60.0, and 51.0\% respectively; $P<0.001$ ). 
Table 3 Clinical data by baseline levels of TSH. Baseline TSH was not measured in 99 patients, there was no record of TSH estimation or otherwise in 20 patients, and baseline levels were not submitted in a further 11 patients.

\begin{tabular}{|c|c|c|c|c|c|}
\hline \multirow[b]{2}{*}{ Clinical data } & \multicolumn{3}{|c|}{ TSH level (mIU/l) } & \multirow[b]{2}{*}{ Total $(n=1125)$} & \multirow[b]{2}{*}{$P$ value } \\
\hline & $<0.2(n=6)$ & $0.2-4(n=53)$ & $>4(n=1066)$ & & \\
\hline \multicolumn{6}{|l|}{ Diagnostic circumstances $^{\mathrm{a}}, n(\%)$} \\
\hline Suspected hypothyroidism & $1(16.7)$ & $29(54.7)$ & $892(83.7)$ & $922(82.0)$ & $<0.001$ \\
\hline Detection of goiter & $1(16.7)$ & $8(15.1)$ & $157(14.7)$ & $166(14.8)$ & NS \\
\hline Other known thyroid disease ${ }^{b}$ & $6(100.0)$ & $25(47.2)$ & $154(14.4)$ & $185(16.4)$ & 0.0057 \\
\hline \multicolumn{6}{|l|}{ Etiology of hypothyroidism, $n(\%)$} \\
\hline Not reported & - & - & 4 & 4 & \\
\hline Autoimmune thyroiditis & $1(16.7)$ & $14(26.4)$ & $703(66.2)$ & $718(64.0)$ & $<0.001$ \\
\hline latrogenic cause ${ }^{c}$ & $5(83.3)$ & $29(54.7)$ & $213(20.1)$ & $247(22.0)$ & $<0.001$ \\
\hline Other non-iatrogenic cause ${ }^{d}$ & - & $10(18.9)$ & $146(13.7)$ & $156(13.9)$ & NS \\
\hline $\begin{array}{l}\text { Mean (S.D.) interval from determination of } \\
\text { TSH to the start of the treatment, days }\end{array}$ & $28.2(35.0)$ & $29.8(30.5)$ & $16.3(22.9)$ & $17.0(23.5)$ & $<0.001$ \\
\hline \multicolumn{6}{|l|}{ Positive for anti-thyroid antibodies (\%) } \\
\hline Anti-thyroperoxidase & 20.0 & 24.5 & 49.1 & 47.8 & 0.0024 \\
\hline Anti-thyroglobulin & 20.0 & 16.7 & 35.9 & 35.0 & 0.0034 \\
\hline
\end{tabular}

NS, not statistically significant.

${ }^{\mathrm{a}}$ More than one response could be given for each patient.

${ }^{\mathrm{b}}$ Thyroid nodular disease.

Includes surgery (38\%), radioiodine therapy (12\%), treatment with anti-thyroid drugs (12\%), combinations of radioiodine therapy and anti-thyroid drugs $(2 \%)$, and other treatments $(36 \%)$.

${ }^{d}$ Causes recorded in more than two patients were 'unknown' (45\%), subclinical hypothyroidism (9\%), investigation ongoing ( $\left.8 \%\right)$, goiter $(6 \%)$, old age $(4 \%)$, thyroid hypoplasia $(3 \%)$, thyroid atrophy $(3 \%)$, pregnancy $(2 \%)$, and family history $(2 \%)$. Causes recorded in one or two patients were: iodine deficiency, emotional shock, craniopharyngioma, central hypothyroidism, multinodular thyroid dystrophy, alcohol and tobacco abuse, hematocele, central hypothyroidism, deficiency-induced hypothyroidism, familial hypothyroidism, chronic kidney disease, pituitary insufficiency, menopause, thyroid nodules, secondary hypothyroidism, primary hypothyroidism, involutional thyroid, thyroiditis, non-autoimmune thyroiditis, de Quervain's subacute thyroiditis, and viral thyroiditis.

\section{Differences in management between PCPs and endocrinologists}

Patients enrolled in the study by PCPs were mostly cared for by the enrolling PCP $(n=786,94.1 \%)$. Threequarters of patients enrolled by endocrinologists had been referred by other physicians $(n=319,76.0 \%)$. Patients with prior thyroid disease were more likely to be diagnosed with hypothyroidism by an endocrinologist than by a PCP. PCPs were more likely than endocrinologists to diagnose patients on the basis of a clinical suspicion of hypothyroidism or the presence of a goiter (Table 1).

One of the largest differences noted between physician groups was in the use of scintigraphy, which was carried out in 180 patients: $151(23.2 \%)$ patients managed by PCPs and $29(9.5 \%)$ patients managed by endocrinologists $(P<0.001)$. Further analysis showed that, among PCPs, scintigraphy was more commonly requested among older physicians ( $>55$ years, who examined $30.6 \%$ of patients), compared with their younger colleagues $(<45$ years, $13.3 \%$ patients; $45-55$ years, $21.6 \%$ patients $)(P=0.0012)$. There was no physician age effect on scintigraphy uptake by endocrinologists. Uptake of thyroid ultrasound was similar in the two groups of physicians (98.3 vs $97.0 \%$ of patients).

$\mathrm{L}-\mathrm{T}_{4}$ was the medication chosen for almost all patients by both PCPs and endocrinologists. Both groups followed similar steps for dose titration. However, endocrinologists were significantly more likely than PCPs to provide counseling for their patients (60.9 vs
$39.7 \%, P<0.001)$. When counseling was offered, endocrinologists gave advice about taking treatment on an empty stomach more often than PCPs (69.1 vs $22.9 \%$ ), but PCPs were more likely to give advice on recognizing the signs of an overdose than endocrinologists (35.6 vs $20.6 \%$ ). There was no significant relationship between the provision of counseling and the measurement of TSH at follow-up.

\section{Discussion}

To the best of our knowledge, no other studies have investigated real-life approaches to the initial management of hypothyroidism in France. The ORCHIDÉE study collected data from PCPs and endocrinologists for 1255 patients, mostly women, with an average age of 50-55 years. This patient population is similar to populations with hypothyroidism reported in other studies from France and the UK $(10,11,15,16)$. The large patient cohort and the profile of the participating physicians, which was largely representative of the wider pool, indicate that these results are likely to be representative of medical practice in France.

In line with earlier reports $(6,7,17,18)$, the most common cause of hypothyroidism was autoimmune thyroiditis, which was reported in $59 \%$ of patients. Iatrogenic causes were the second most frequent category, in almost $28 \%$ of patients, largely owing to thyroidectomy. Iatrogenic causes were reported with an incidence rate of only $1.8 \%$ in a Danish 
population-based study (19). Although this latter study excluded hypothyroidism caused by previous treatment of hyperthyroidism, this large difference between our results suggests that thyroidectomy may play a larger part in the management of thyroid diseases in France than in Denmark, and raises questions about medical strategies in the two countries.

There was good uptake of the major diagnostic techniques across both groups of physicians. Measurement of TSH is recommended by guidelines as the primary diagnostic test for hypothyroidism $(5,20)$, and was undertaken in $90 \%$ of patients in the current study. Over half of the patients had serum TSH levels of $<10 \mathrm{mIU} / \mathrm{l}$ and thus warranted additional investigation, which may explain the relatively high rates of $\mathrm{FT}_{4}$ measurement. Patients with hypothyroidism as a consequence of treatment for hyperthyroidism were less likely to have either $\mathrm{TSH}$ or $\mathrm{FT}_{4}$ levels measured before starting the treatment than patients with other diagnoses.

Sonography was used in almost every case of spontaneous hypothyroidism but is recommended only for showing or confirming the presence of nodules, for measuring thyroid volume, or for demonstrating reduced echogenicity in autoimmune thyroiditis (20). Thus, the use of thyroid imaging in over $75 \%$ of patients in this study is high, and its value in patient management may be questionable in some of the cases included.

The apparent excessive use of scintigraphy by PCPs, particularly among older practitioners, suggests that this group may have little awareness of current guidelines and is of concern, since inappropriate use of scintigraphy exposes patients to radiation for no clinical benefit.

Treatment of hypothyroidism by PCPs and endocrinologists was mostly in line with current French recommendations $(20,21)$. $\mathrm{L}^{-} \mathrm{T}_{4}$ was prescribed in almost all patients $(98 \%)$, with a mean interval between dose escalations of 6 weeks. This practice is largely in line with the guidance for a population with a relatively low risk for cardiovascular disease, although the dose could have been increased more rapidly in such a low-risk population. It should also be noted that, in the follow-up, $\mathrm{FT}_{4}$ levels were frequently assessed, even though TSH levels are the only necessary measurement in this context.

Repeat TSH data were available from almost $95 \%$ of patients in whom follow-up data were available, and the results show a major reduction in mean TSH levels, although the average dose of $\mathrm{L}-\mathrm{T}_{4}$ was below the recommended level $(\geq 1.7 \mu \mathrm{g} / \mathrm{kg})$ for full thyroid hormone replacement (11).

The ORCHIDÉE study was designed to be an observational study and not to alter the regular practice of the physicians taking part. As for any observational study, it relies on the reports made by the participating physicians without independent verification. In reporting this study, we have followed the Strengthening the Reporting of Observational Studies in Epidemiology (STROBE) guidelines (http://www.strobe-statement.org/) to ensure that it is reported as fully and accurately as possible.

Although physicians were invited to be involved in this study using a random selection approach, the physicians who responded positively were self-selecting and differed in some respects from the overall PCP and endocrinologist populations. The study was also limited by the broad inclusion criteria, which permitted the inclusion of patients with a wide variety of conditions resulting in hypothyroidism. However, the use of the broad inclusion criteria can also be considered as a strength, as it allowed the study to reflect the management of a real-life cohort of patients with a new diagnosis of hypothyroidism.

\section{Conclusions}

The ORCHIDÉE study provides valuable information about the real-life clinical management of hypothyroidism in France. The large and representative newly diagnosed patient population and the inclusion of cohorts managed by both PCPs and endocrinologists make the findings robust and probably representative of the wider hypothyroid population in France. The results show a high level of awareness of, and willingness to treat, subclinical hypothyroidism; indeed, about half of the patients being treated with $\mathrm{L}-\mathrm{T}_{4}$ had presented with relatively mild hypothyroidism. Both groups of physicians used TSH estimation extensively as the basis for diagnosis and monitoring, and there was also widespread use of $\mathrm{FT}_{4}$ levels, thyroid antibodies, and sonography. Although this study provides reassurance that patients are largely being treated in accordance with guidelines by endocrinologists and PCPs in France, it identified some areas for improvement, which could potentially improve the cost-effectiveness of routine practice.

\section{Declaration of interest}

B Delemer has been an invited speaker at Merck Serono-sponsored congresses. J-P Aubert was a consultant for the ORCHIDÉE study. P Nys has been an invited speaker at Merck Serono-sponsored congresses. F Landron is an employee of Merck Serono S.A.S. S Bouée is a statistical consultant for Merck Serono.

\section{Funding}

This study was supported by Merck Serono S.A.S. Lyon, France, an affiliate of Merck KGaA, Darmstadt, Germany.

\section{Author contribution statement}

B Delemer and S Bouée were involved in the study design, data interpretation, drafting, and reviewing the manuscript. J-P Aubert and $P$ Nys were involved in the study design, data interpretation, and reviewing the manuscript. F Landron coordinated the study and was involved in the data interpretation, drafting, and reviewing the manuscript. 


\section{Acknowledgements}

The authors take full responsibility for the content of the article and thank Joanna Wright, DPhil (Caudex Medical, Oxford, UK, supported by Merck Serono S.A., Geneva, a branch of Merck Serono S.A., Coinsins, Switzerland, an affiliate of Merck KGaA, Darmstadt, Germany) for assistance in preparing the outline and initial draft of the manuscript, including preparation of tables, and collating the comments of authors and other named contributors.

\section{References}

1 Caldwell G, Kellett HA, Gow SM, Beckett GJ, Sweeting VM, Seth J \& Toft AD. A new strategy for thyroid function testing. Lancet 19851 1117-1119. (doi:10.1016/S0140-6736(85)92429-8)

2 Klee GG \& Hay ID. Biochemical testing of thyroid function. Endocrinology and Metabolism Clinics of North America 199726 763-775. (doi:10.1016/S0889-8529(05)70281-4)

3 Weetman AP. Hypothyroidism: screening and subclinical disease. BMJ 1997314 1175-1178. (doi:10.1136/bmj.314.7088.1175)

4 Karmisholt J, Andersen S \& Laurberg P. Variation in thyroid function in subclinical hypothyroidism: importance of clinical follow-up and therapy. European Journal of Endocrinology 2011164 317-323. (doi:10.1530/EJE-10-1021)

5 Baskin HJ, Cobin RH, Duick DS, Gharib H, Guttler RB, Kaplan MM \& Segal RL. American Association of Clinical Endocrinologists medical guidelines for clinical practice for the evaluation and treatment of hyperthyroidism and hypothyroidism. Endocrine Practice 20028 457-469.

6 Vaidya B \& Pearce SH. Management of hypothyroidism in adults. BMJ 2008337 a801. (doi:10.1136/bmj.a801)

7 Diez JJ. Hypothyroidism in patients older than 55 years: an analysis of the etiology and assessment of the effectiveness of therapy. Journals of Gerontology Series A: Biological Sciences and Medical Sciences 200257 M315-M320. (doi:10.1093/gerona/57. 5.M315)

8 Topliss DJ \& Eastman CJ. 5: Diagnosis and management of hyperthyroidism and hypothyroidism. Medical Journal of Australia $2004180186-193$.

9 Knudsen N, Jorgensen T, Rasmussen S, Christiansen E \& Perrild H. The prevalence of thyroid dysfunction in a population with borderline iodine deficiency. Clinical Endocrinology 199951 361-367. (doi:10.1046/j.1365-2265.1999.00816.x)

10 Vanderpump MP, Tunbridge WM, French JM, Appleton D, Bates D, Clark F, Grimley EJ, Hasan DM, Rodgers H, Tunbridge F et al. The incidence of thyroid disorders in the community: a twenty-year follow-up of the Whickham Survey. Clinical Endocrinology 199543 55-68. (doi:10.1111/j.1365-2265.1995.tb01894.x)

11 Valeix P, Dos SC, Castetbon K, Bertrais S, Cousty C \& Hercberg S. Thyroid hormone levels and thyroid dysfunction of French adults participating in the SU.VI.MAX study. Annals of Endocrinology 200465 477-486. (doi:10.1016/S0003-4266(04)95956-2)

12 Mandel SJ, Brent GA \& Larsen PR. Levothyroxine therapy in patients with thyroid disease. Annals of Internal Medicine 1993 $119492-502$.

13 Agence Nationale d'Accréditation et d'Evaluation en Sante. Diagnostic et surveillance biologique de l'hypothyroïdie de l'adulte 1998. Available at: http://www.has-sante.fr/portail/upload/docs/ application/pdf/hyperthy_rap.pdf. Last accessed: 17 April 2012.

14 Falissard B. Analysis of questionnaire Data with $R$, Chapman and Hall edn. CRC press Taylor, 2011.

15 Nys P, Cordray JP \& Merceron RE. Etiologic discussion and clinical relevance of thyroid ultrasonography in subclinical hypothyroidism. A retrospective study in 1845 patients. Annals of Endocrinology 200970 59-63. (doi:10.1016/j.ando.2008.09.009)

16 Tunbridge WM, Evered DC, Hall R, Appleton D, Brewis M, Clark F, Evans JG, Young E, Bird T \& Smith PA. The spectrum of thyroid disease in a community: the Whickham survey. Clinical Endocrinology $1977 \quad 7$ 481-493. (doi:10.1111/j.1365-2265.1977. tb01340.x)

17 Orgiazzi J \& Bournaud C. Current views on hypothyroidism in adults. La Revue du Praticien 200555 158-166.

18 Chanson P. Hypothyroidism in adults. La Revue du Praticien 2006 56 1369-1375.

19 Carle A, Laurberg P, Pedersen IB, Knudsen N, Perrild H, Ovesen L, Rasmussen LB \& Jorgensen T. Epidemiology of subtypes of hypothyroidism in Denmark. European Journal of Endocrinology 2006154 21-28. (doi:10.1530/eje.1.02068)

20 Item 248: Hypothyroidie. 2004 Available at: http://www. endocrino.net/download/DCEM/248_1204.pdf. Last accessed: 17 April 2012.

21 Société Française de Radiologie (SFR) and Société Française de Biophysique et de Médecine Nucléaire (SFBMN). Guide du bon usage des examens d'imagerie médicale 2005.

Received 30 November 2011

Revised version received 5 September 2012

Accepted 3 October 2012 